\title{
Laboratory Predictors of COVID-19 Mortality: A Retrospective Analysis from Tongji Hospital in Wuhan
}

\author{
Ting Zheng $\mathbb{D}^{1,2}$ Xinxin Liu, ${ }^{2}$ Yingying Wei, ${ }^{2}$ Xinlu Li, ${ }^{2}$ Bing Zheng, ${ }^{1}$ Quan Gong $\mathbb{D},{ }^{1}$ \\ Lingli Dong $\mathbb{D}^{2}{ }^{2}$ and Jixin Zhong $\mathbb{B}^{2}$ \\ ${ }^{1}$ Department of Immunology, School of Medicine, Yangtze University, Jingzhou, Hubei 434023, China \\ ${ }^{2}$ Department of Rheumatology and Immunology, Tongji Hospital, Tongji Medical College, Huazhong University of Science \\ and Technology, Wuhan, Hubei 430030, China
}

Correspondence should be addressed to Quan Gong; gongquan1998@163.com, Lingli Dong; tjhdongll@163.com, and Jixin Zhong; zhongjixin620@163.com

Received 7 December 2020; Accepted 16 February 2021; Published 24 February 2021

Academic Editor: Mirella Giovarelli

Copyright (C) 2021 Ting Zheng et al. This is an open access article distributed under the Creative Commons Attribution License, which permits unrestricted use, distribution, and reproduction in any medium, provided the original work is properly cited.

Background. Novel coronavirus disease 2019 (COVID-19), an acute respiratory disease caused by severe acute respiratory syndrome coronavirus 2 (SARS-CoV-2), rapidly progressed to a global pandemic. Currently, there are limited effective medications approved for this deadly disease. Objective. To investigate the potential predictors of COVID-19 mortality and risk factors for hyperinflammation in COVID-19. Methods. Retrospective analysis was carried out in 1,149 patients diagnosed with COVID-19 in Tongi Hospital, Wuhan, China, from 1/13/2020 to 3/15/2020. Results. We found significant differences in the rates of hyperuricemia (OR: 3.17, 95\% CI: 2.13-4.70; $p<0.001$ ) and hypoalbuminemia (OR: 5.68, 95\% CI: 3.97-8.32; $p<0.001$ ) between deceased and recovered patients. The percentages of hyperuricemia in deceased patients and recovered patients were $23.6 \%$ and $8.9 \%$, respectively, which were higher than the reported age-standardized prevalence of $6.2 \%$ in Chinese population. Of note, the percentages of both IL-6 and uric acid levels in survived COVID-19 patients were above 90\%, suggesting that they might be good specificity for indicators of mortality in COVID-19 patients. The serum level of uric acid (UA) was positively associated with ferritin, TNF- $\alpha$, and IL- 6 but not with anti-inflammatory cytokine IL-10. In addition, the levels of these proinflammatory cytokines in COVID-19 patients showed a trend of reduction after uric acid lowering therapy. Conclusions. Our results suggest that uric acid, the end product of purine metabolism, was increased in deceased patients with COVID-19. In addition, the serum level of uric acid was positively associated with inflammatory markers. Uric acid lowering therapy in COVID-19 patients with hyperuricemia may be beneficial.

\section{Introduction}

Coronavirus disease 19 (COVID-19) caused by the ribonucleic acid (RNA) virus severe acute respiratory syndrome coronavirus 2 (SARS-CoV-2), emerged in Wuhan City, Hubei Province, China, has spread rapidly across the world. At the time of drafting this manuscript (Oct. 19, 2020), the worldwide death toll from the COVID-19 pneumonia eclipsed 1,000,000 [1], and the number of people infected continues to slowly climbed upward. Although predictors such as high-sensitivity C-reactive protein (hsCRP), aspartate aminotransferase (AST), and D-dimer for mortality of COVID-19 patients had been determined [2], more risk predictors and prognostic factors still desperately needed to been found to improve the treatment programs for infected patients, especially for patients with other underlying diseases (such as identified risk factors indicator cardiac troponin I to preexisting concurrent cardiovascular or cerebrovascular diseases [3]; BMI for COVID-19 severity in the population living with diabetes in hospital admission [4]). 

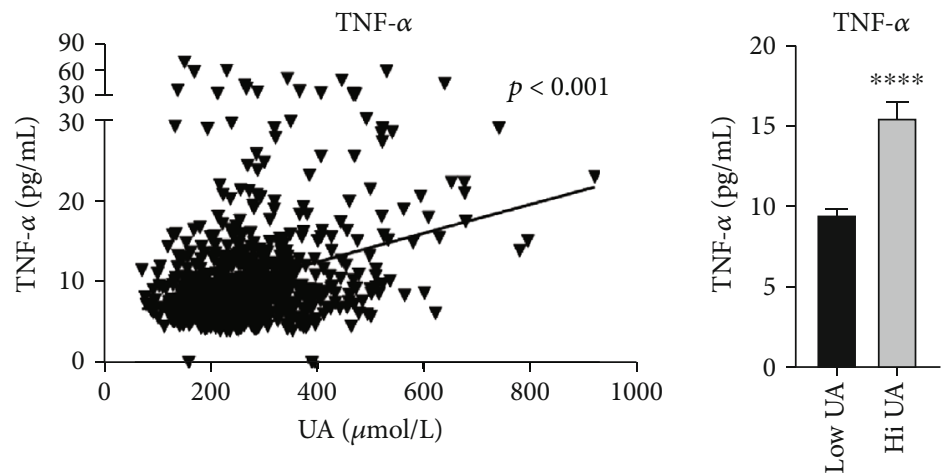

(a)
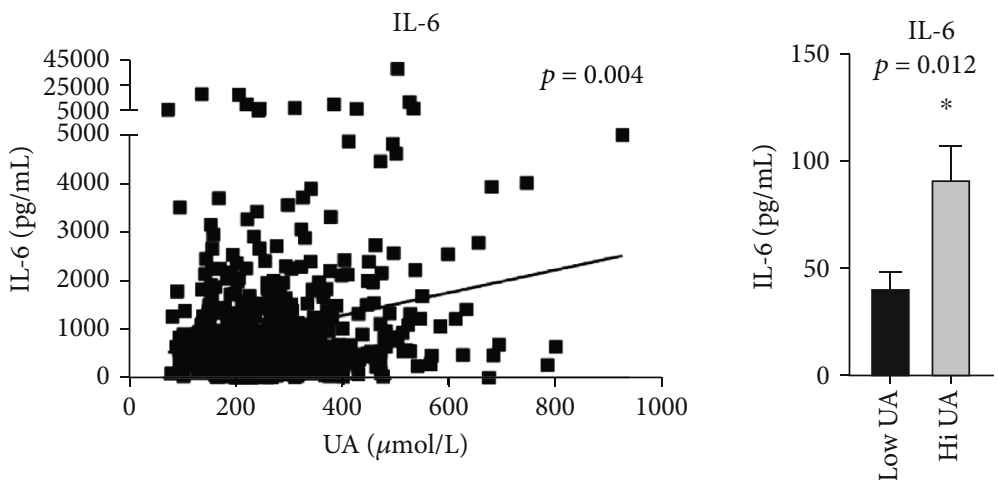

(b)
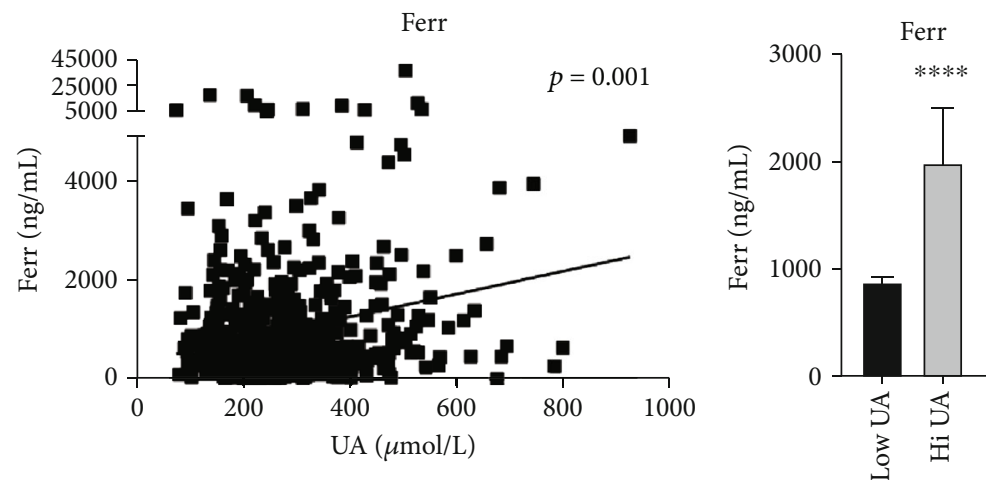

(c)
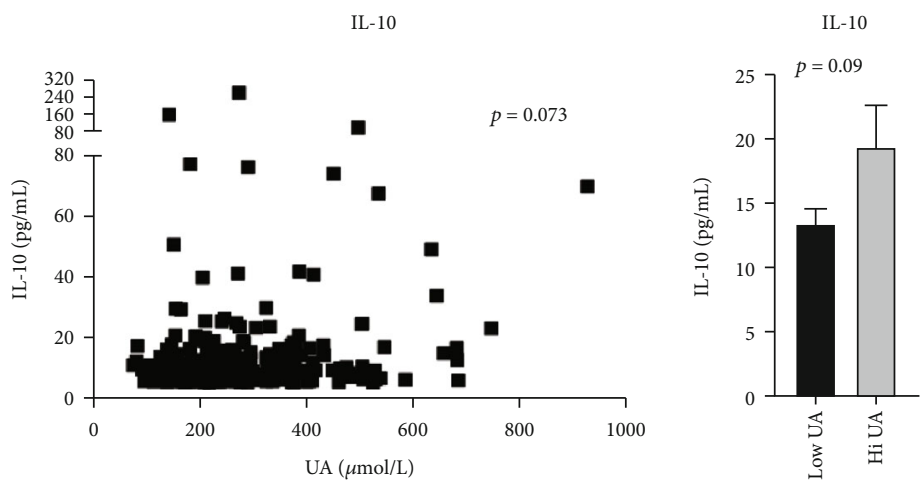

(d)

FIgUre 1: Continued. 


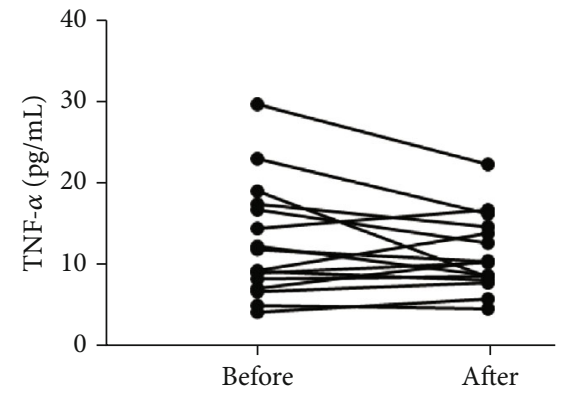

(e)

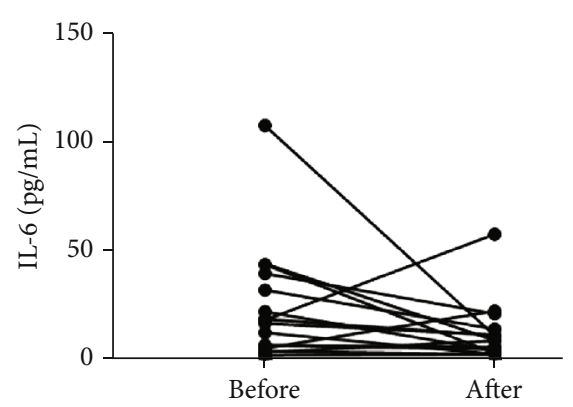

$(\mathrm{f})$

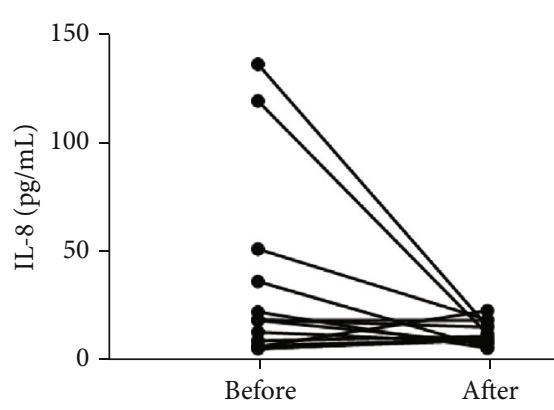

(g)

FIGURE 1: Uric acid is associated with serum levels of TNF- $\alpha$, IL-6, and ferritin in COVID-19 patients. (a) Left, serum uric acid (UA) is associated with serum TNF- $\alpha$ level; right, serum TNF- $\alpha$ level in patients with a UA level of lower or higher than $400 \mu \mathrm{mol} / \mathrm{L}$. (b) Left, association between serum UA and IL-6 levels; right, serum IL-6 level in patients with a UA level of lower or higher than $400 \mu \mathrm{mol} / \mathrm{L}$. (c) Left, association between serum UA and ferritin levels; right, serum ferritin level in patients with a UA level of lower or higher than $400 \mu \mathrm{mol} / \mathrm{L}$. (d) Left, association between serum UA and IL-10 levels; right, serum IL-10 level in patients with a UA level of lower or higher than $400 \mu \mathrm{mol} / \mathrm{L}$. (e) Serum TNF- $\alpha$ level before and after uric acid lowering therapy. (f) Serum IL-6 level before and after uric acid lowering therapy. (g) Serum IL-8 level before and after uric acid lowering therapy.

\section{Materials and Methods}

2.1. Participants and Clinical Variables. We analyzed and observed serological tests result a number of laboratory parameters may serve as predictors of disease progression in 1,149 in patients diagnosed with COVID-19 in Tongji Hospital, Wuhan, China, from $1 / 13 / 2020$ to $3 / 15 / 2020$ and performed statistical analysis to estimate ORs and 95\% CIs of mortality. The correlation between serum uric acid and other inflammatory factors and the content of these inflammatory factors before and after treatment were compared.

2.2. Statistical Analysis. Categorical variables were reported as percentages. Combined odds ratios (ORs) and 95\% CIs were evaluated as effect size using uni- and multivariate analyses. We used linear regression to evaluate any association between two variables. $p$ values less than 0.05 were considered statistically significant.

2.3. Ethics. The study was performed in accordance with the ethical standards laid down in the Declaration of Helsinki. Our work has been reviewed and approved by Tongji Hospital Ethics Committee.

\section{Results}

In univariate analyses, patients who died from COVID-19 had higher hyperinflammation markers than patients who survived: lactate dehydrogenase (LDH, OR: 25.14, 95\% CI: 17.06-37.53; $p<0.0001$ ), AST (OR: 5.08, 95\% CI: 3.67-7.05; $<0.0001$ ), alanine aminotransferase (ALT, OR: 1.52, 95\% CI: $1.07-2.14 ; p=0.018$ ), ferritin (OR: 12.92 , 95\% CI: $5.74-$ $37.0 ; p<0.0001$ ), and inflammatory cytokines TNF- $\alpha$ (OR: 4.34, 95\% CI: 2.90-6.59; $p<0.0001$ ), Interleukin-6 (IL-6, OR: $68.63,95 \%$ CI: 31.02-182.30; $p<0.0001)$, Interleukin-8 (IL-8, OR: 7.60, 95\% CI: 4.26-13.80; $p<0.0001$ ), and antiinflammatory cytokines Interleukin-10 (IL-10, OR: 8.06, 95\% CI: $3.56-20.75 ; p<0.0001)$. In addition to these previously confirmed indicators of COVID-19 disease severity
$[5,6]$, we found significant differences in the rates of hyperuricemia (OR: 3.17, 95\% CI: 2.13-4.70; $p<0.001$ ) and hypoalbuminemia (OR: 5.68, 95\% CI: 3.97-8.32; $p<0.001$ ) between deceased and recovered patients. The percentages of hyperuricemia in deceased patients and recovered patients were $23.6 \%$ and $8.9 \%$, respectively, which are higher than the reported age-standardized prevalence of $6.2 \%$ in Chinese population [7]. Of note, the percentages of both IL- 6 and uric acid (UA) levels in survived COVID-19 patients were above $90 \%$, suggesting that these two factors have a good specificity when used as indicators for COVID-19 mortality.

Interestingly, the serum level of uric acid is positively associated with ferritin, TNF- $\alpha$, and IL- 6 but not with antiinflammatory cytokine IL-10 (Figures $1(\mathrm{a})-1(\mathrm{~d})$ ). Patients with a serum uric acid level over $400 \mu \mathrm{mol} / \mathrm{L}$ had higher serum levels of TNF- $\alpha$, IL-6, and ferritin. To investigate if the increase of uric acid contributes to the hyperinflammatory status in COVID-19, we analyzed cytokine levels before and after administration of uric acid lowering agents (febuxostat or benzbromarone). A total of 16 COVID-19 patients with an acute gout attack during the hospital stay were examined for cytokine profile before and after the treatment with uric acid lowering therapy. After an average of 7 days of therapy, there was a trend toward reduced serum levels of IL-6, IL-8, and TNF- $\alpha$ (Figures $1(\mathrm{e})-1(\mathrm{~g})$ ). There were 9 out of the 16 patients taking uric acid lowering agents that had a serum uric acid level over $400 \mu \mathrm{mol} / \mathrm{L}$. While 8 out of these 9 patients recovered from COVID-19, only 74 out of the 121 hyperuricemia patients without uric acid lowering agents survived ( $88.9 \%$ vs. $61.2 \% ; p=0.096)$.

\section{Discussion}

We observed in this study that deceased COVID-19 patients had a higher rate of hyperuricemia. Given the fact that biomarkers of tissue damage such as LDH, AST, ALT, and ferritin were increased in severely ill or deceased COVID-19 patients (Table 1 and references $[5,6]$ ), increased percentages 
TABLE 1: Laboratory parameters in 1,149 COVID-19 patients hospitalized in Tongji hospital between 1/13/2020 and 3/15/2020.

\begin{tabular}{|c|c|c|c|c|}
\hline & Deceased, $n(\%)$ & Survived, $n(\%)$ & OR $(95 \% \mathrm{CI})$ & $p$ \\
\hline \multicolumn{5}{|l|}{ Age (years) } \\
\hline$<60, n(\%)$ & $41(19.0)$ & $522(55.9)$ & 1.00 & - \\
\hline$\geq 60, n(\%)$ & $175(81.0)$ & $411(44.1)$ & $3.36(2.36-4.89)$ & $<0.0001$ \\
\hline \multicolumn{5}{|l|}{ Gender } \\
\hline Male, $n(\%)$ & $145(67.1)$ & 477 (47.9) & $2.23(1.63-3.09)$ & $<0.0001$ \\
\hline Female, $n(\%)$ & $71(32.9)$ & $486(52.1)$ & 1.00 & - \\
\hline \multicolumn{5}{|l|}{ IL-6 (pg/mL) } \\
\hline$>150, n(\%)$ & $61(43.3)$ & $6(1.1)$ & $68.63(31.02-182.30)$ & $<0.0001$ \\
\hline$\leq 150, n(\%)$ & $80(56.7)$ & $540(98.9)$ & 1.00 & - \\
\hline \multicolumn{5}{|l|}{ IL-10 (pg/mL) } \\
\hline$>15, n(\%)$ & $72(68.6)$ & $44(29.7)$ & $8.06(3.56-20.75)$ & $<0.0001$ \\
\hline$\leq 15, n(\%)$ & $33(31.4)$ & $104(70.3)$ & 1.00 & - \\
\hline \multicolumn{5}{|l|}{ IL-8 (pg/mL) } \\
\hline$>100, n(\%)$ & $32(24.8)$ & $22(4.2)$ & $7.60(4.26-13.80)$ & $<0.0001$ \\
\hline$\leq 100, n(\%)$ & $97(75.2)$ & $507(95.8)$ & 1.00 & - \\
\hline \multicolumn{5}{|l|}{ TNF- $\alpha(\mathrm{pg} / \mathrm{mL})$} \\
\hline$>100, n(\%)$ & $83(65.4)$ & $159(30.3)$ & $4.34(2.90-6.59)$ & $<0.0001$ \\
\hline$\leq 100, n(\%)$ & $44(34.6)$ & $366(69.7)$ & 1.00 & - \\
\hline \multicolumn{5}{|l|}{ Uric acid $(\mu \mathrm{mol} / \mathrm{L})$} \\
\hline$>400, n(\%)$ & $48(23.6)$ & $82(8.9)$ & $3.17(2.13-4.70)$ & $<0.0001$ \\
\hline$\leq 400, n(\%)$ & $155(76.4)$ & $840(91.1)$ & 1.00 & - \\
\hline \multicolumn{5}{|l|}{ Ferritin (ng/mL) } \\
\hline$>400(\mathrm{M}) / 300(\mathrm{~F}), n(\%)$ & $123(96.1)$ & $337(65.6)$ & $12.92(5.74-37.0)$ & $<0.0001$ \\
\hline$\leq 400(\mathrm{M}) / 300(\mathrm{~F}), n(\%)$ & $5(3.9)$ & $177(34.4)$ & 1.00 & - \\
\hline \multicolumn{5}{|l|}{ Albumin (g/L) } \\
\hline$\geq 35, n(\%)$ & $40(19.9)$ & $537(58.4)$ & 1.00 & - \\
\hline$<35, n(\%)$ & $161(80.1)$ & $382(41.6)$ & $5.68(3.97-8.32)$ & $<0.0001$ \\
\hline \multicolumn{5}{|l|}{ LDH (U/L) } \\
\hline$>450, n(\%)$ & $123(62.4)$ & $57(6.2)$ & $25.14(17.06-37.53)$ & $<0.0001$ \\
\hline$\leq 450, n(\%)$ & $74(34.6)$ & $862(93.8)$ & 1.00 & - \\
\hline \multicolumn{5}{|l|}{$\operatorname{ALT}(\mathrm{U} / \mathrm{L})$} \\
\hline$>40, n(\%)$ & $57(28.4)$ & $189(20.6)$ & $1.52(1.07-2.14)$ & 0.018 \\
\hline$\leq 40, n(\%)$ & $144(71.6)$ & $728(79.4)$ & 1.00 & - \\
\hline \multicolumn{5}{|l|}{ AST (U/L) } \\
\hline$>40, n(\%)$ & $102(50.7)$ & $153(16.7)$ & $5.08(3.67-7.05)$ & $<0.0001$ \\
\hline$\leq 40, n(\%)$ & $99(49.3)$ & $764(83.3)$ & 1.00 & - \\
\hline \multicolumn{5}{|l|}{ AST/ALT } \\
\hline$>1, n(\%)$ & $159(79.1)$ & $533(60.3)$ & $2.48(1.74-3.61)$ & $<0.0001$ \\
\hline$\leq 1, n(\%)$ & $42(20.9)$ & $364(39.7)$ & 1.00 & - \\
\hline
\end{tabular}

ALT: alanine aminotransferase; AST: aspartate aminotransferase; IL-6: interleukin 6; IL-8: interleukin 8; IL-10: interleukin 10; LDH: lactate dehydrogenase; TNF- $\alpha$ : tumor necrosis factor $\alpha$.

of hyperuricemia in COVID-19 patients, especially in deceased patients, may be a result of tissue damage/cell death. Uric acid has been characterized as a danger signal to alarm immune system to cell injury and initiate immune responses to clear damaged cells/tissues [8]. Uric acid is a degradation product of purines and presents in the blood at a high concentration approaching to the saturation threshold. In the event of tissue damage, intracellular stores of uric acid are released out of the cells, which may cause hyperuricemia and form crystals. Crystallized form of uric acid is a strong inducer of NLRP3 inflammasome, inciting robust inflammation. Nucleated uric acid is able to stimulate the production of inflammatory cytokines such as IL-1, TNF- $\alpha$, and IL- 6 in gout, a classic disease caused by hyperuricemia. In this case series, we observed positive correlations between uric acid and inflammatory markers in patients with COVID-19. In 
addition, uric acid lowering treatment with febuxostat and/or benzbromarone reduced levels of proinflammatory cytokines in COVID-19 patients. There was also a trend toward reduced mortality rate in hyperuricemia patients with uric acid lowering therapy. These results suggest that uric acid lowering therapy may be beneficial in COVID-19 patients with hyperuricemia. However, a further investigation with larger sample size and randomized controls is required to confirm the beneficial effects of uric acid lowering therapy in reducing proinflammatory cytokines.

In summary, uric acid released from the injured tissues/ cells may serve as a danger signal to amplify the hyperinflammatory response in severe COVID-19 cases. Uric acid lowering therapy in COVID-19 patients with hyperuricemia patients may be able beneficial.

\section{Data Availability}

Derived data supporting the findings of this study are available from the corresponding author (JZ, LD, QG) on request.

\section{Conflicts of Interest}

The authors declare no conflicts of interest.

\section{Authors' Contributions}

All the authors contributed substantially to the work. Drs. Zhong, Dong, and Gong contributed equally to this article.

\section{Acknowledgments}

This work was supported by the National Natural Science Foundation of China (grant numbers 81974254, 81670431, and 81771754) and the Tongji Hospital Clinical Research Flagship Program (grant number no. 2019CR206).

\section{References}

[1] "WHO coronavirus disease (COVID-19) dashboard," October 2020, https://covid19. who.int/?gclid=CjwKCAjwq832BRA5E iwACvCWsUXTxEZePK3xTjFXswIn7L0b_rm1mt5LGtFld8q Q3YKGqVk_I159qhoCyxcQAvD_BwE.

[2] K. Wang, P. Zuo, Y. Liu et al., "Clinical and laboratory predictors of in-hospital mortality in patients with coronavirus disease-2019: a cohort study in Wuhan, China," Clinical infectious diseases, vol. 71, no. 16, pp. 2079-2088, 2020.

[3] L. L. R. Du RH, C. Q. Yang, W. Wang et al., "Predictors of mortality for patients with COVID-19 pneumonia caused by SARS-CoV-2: a prospective cohort study," European Respiratory Journal, vol. 55, no. 5, article 2000524, 2020.

[4] B. Cariou, S. Hadjadj, M. Wargny et al., "Phenotypic characteristics and prognosis of inpatients with COVID-19 and diabetes: the CORONADO study," Diabetologia, vol. 63, no. 8, pp. 15001515, 2020.

[5] C. Huang, Y. Wang, X. Li et al., "Clinical features of patients infected with 2019 novel coronavirus in Wuhan, China," Lancet, vol. 395, no. 10223, pp. 497-506, 2020.

[6] D. Wang, B. Hu, C. Hu et al., "Clinical characteristics of 138 hospitalized patients with 2019 novel coronavirus-infected pneumonia in Wuhan, China," JAMA, vol. 323, no. 11 , pp. 1061-1069, 2020.

[7] P. Song, H. Wang, W. Xia, X. Chang, M. Wang, and L. An, "Prevalence and correlates of hyperuricemia in the middleaged and older adults in China," Scientific reports, vol. 8, no. 1, article 4314, 2018.

[8] Y. Shi, J. E. Evans, and K. L. Rock, "Molecular identification of a danger signal that alerts the immune system to dying cells," Nature, vol. 425, no. 6957, pp. 516-521, 2003. 